\title{
Covid 19
}

blog.kulturwissenschaften.de/covid-19/

Christian Jaser Mieke Roscher

\section{Eine tiergeschichtliche Annäherung an eine Pandemie}

Ist das Coronavirus tatsächlich eine ,Revenge of the Pangolins', ein Racheakt der vom Aussterben bedrohten Schuppentiere gegen den Menschen? ${ }^{1}$ Gegenwärtig gilt ein sog. wet market in der chinesischen Stadt Wuhan als Ausgangspunkt der SARS-CoV-2Infektion, genauer der dortige Handel mit Wildtieren und deren unkontrollierte Schlachtung. Damit werden im Kern die Entstehung und Ausbreitung des Coronavirus auf eine neue, ökologisch höchst problematische Intensitätsstufe der Mensch-Tier-Beziehungen zurückgeführt, nämlich auf den immer engeren Kontakt von immer mehr Menschen mit Wildtieren, begünstigt durch das Vordringen des Menschen auch in entlegene Regionen und den globalen Tierhandel.

Insofern führt die aktuelle COVID-19-Pandemie mit ihren weltweiten gesundheitspolitischen, wirtschaftlichen, sozialen und mentalen Auswirkungen abermals vor Augen, wie schnell nicht-menschliche Tiere als Krankheitsüberträger und Zwischenwirte in das Zentrum der epidemiologischen Ursachenforschung rücken. Zugleich stellt sich dabei die Frage nach der tierlichen agency im pandemischen Ausbruchsgeschehen. Wo auch immer in der langen Kette von Mensch-Tier-Beziehungen der Urgrund der aktuellen Pandemie aufzufinden sein wird: Epidemien und Pandemien sind Dreh- und Angelpunkte in der zeitspezifischen Reflexion über das jeweilige Verhältnis von humanen und nicht-humanen Aktanten bzw. Akteuren. Angesichts von Infektions- und Gesundheitskrisen entfalten sich um die Koexistenz und Interaktion von Menschen und Tieren medizinische und gesundheitspolitische Aufmerksamkeitsökonomien, aber auch kulturspezifische Deutungs- und Interpretationsspielräume.

\section{Zootiere}

Derartige kulturspezifische Deutungsmuster zeigten sich etwa, als Anfang April 2020 Nadia, eine Tigerin im New Yorker Bronx Zoo, an Corona erkrankte. ${ }^{2}$ Das amerikanische Center of Disease Control war bemüht herauszustreichen, dass sie und auch weitere Großkatzen sich zwar bei einem Tierpfleger angesteckt hätten, auch respiratorische Symptom zeigen würden, jedoch insgesamt die Erkrankung harmlos verlaufen sei. ${ }^{3}$ Anders als andere Wildtiere, die potenziell als Verursacher*innen gebrandmarkt wurden, werden Zootiere als Teile des zivilisatorischen Ganzen gedeutet. Zoologische Gärten als jene Einrichtungen, die als Erben europäischkolonialistischer Zivilisierungsmissionen das Exotische räumlich einzuhegen suchten und heutzutage unter der Fahne des Artenschutzes wiederum, und durchaus neo- 
kolonialistisch, nach dem Erhalt eines arkadischen Anderswo streben, müssen sowohl sicherstellen, dass von den Tieren keine Gefahr ausgeht, aber eben auch, dass sie selbst nicht in Gefahr sind. Die politische Metaphorik, die über Pandemien und Seuchen stets vor allem die Exklusion des (politisch, ethnischen) Anderen artikuliert, hat damit einen semantischen Gegenpol. Auch die politische Metaphorik der Kontrolle wird damit nach wie vor über den Körper von Tieren exerziert. Im Blickpunkt dieses Hygieneregimes standen dabei von Beginn der Pandemie die Menschenaffen, die aufgrund ihrer genetischen Nähe zum Menschen besonders gefährdet seien. Die Tierschutzvereinigung PETA verlangte deshalb nach der Umsetzung eines weitreichenden Hygienekonzeptes für den Schutz der Primaten im Zoo. 4

Gleichzeitig tauchte während des Lockdowns im Frühjahr 2020 eine argumentative Linie auf, die bereits in anderen Krisenzeiten beobachtet werden konnte. Die Zootiere seien einsam ohne die Tausende von Menschen, die größere Tierparks täglich besuchen, und würden sich langweilen. ${ }^{5}$ Bereits während des Zweiten Weltkriegs wurde Normalität anhand der routinemäßigen Zurschaustellung von Tieren signalisiert, weswegen beispielsweise der Londoner Zoo bis auf zwei Wochen zu Beginn des Krieges selbst während des Blitzes geöffnet blieb. ${ }^{6}$ Auch hier wurde der Zoo nicht als einseitiger Ort menschlicher Blicke auf das Tier gezeichnet, sondern als ein interspezifischer Raum, in dem Tiere aktiv an kulturellen Sinnzusammenhängen mitwirken.

\section{Haustiere}

Die Pandemie geht allerdings nicht nur ursächlich auf das Nahverhältnis von Menschen und (Wild-)Tieren zurück, sondern stiftet ihrerseits wiederum vielfach neue human-animalische Beziehungsnetze. Denn im Zuge mannigfaltig verhängter Ausgangs- und Kontaktbeschränkungen stieg die Nachfrage nach Haustieren steil an. ${ }^{7}$ Dieser Umstand lässt sich unschwer aus den spezifischen Bedingungen der Lockdowns und Isolationsgebote erklären: Homeoffice und Haustier gehören nicht nur semantisch zusammen und verweisen gegenwärtig auf eine pandemisch erzwungene Häuslichkeit, die nach tierlichen Begleitern geradezu verlangt: Als Zeitvertreib gegen die Einsamkeit bei stark reduzierten sozialen Kontakten oder als Beschäftigungsoption für Kinder jenseits des home schooling. In Australien, Europa und den Vereinigten Staaten explodierte geradezu die Nachfrage nach Welpen, Erstausstattungen und Hundeversicherungen. Die steigenden Preise für Welpen füllen nicht nur die Kassen von Züchtern und Tierhandlungen, sondern führen auch zu einem teils zwielichtigen Schwarzmarkt auf diversen Onlineplattformen, inklusive falsch deklarierter, kranker und verwahrloster Angebote.

Ein weiterer, schon länger bestehender Haustiertrend nahm in Corona-Zeiten nochmals deutlich an Fahrt auf: die private Nachfrage nach jungen Legehennen, die sich auf diese Weise vom agrarischen Nutztier in ein companion animal verwandeln, das im Garten oder auf dem Balkon gehalten wird. Inmitten von strukturellen Lebensmittelskandalen und situativen Hamsterkäufen (no pun intended) lockt hier 
ein Stück Selbstversorgungsautonomie, für andere wiederum tut sich der Sehnsuchtsort einer weichgezeichneten, wildromantischen Kindheit auf dem Land auf. ${ }^{8}$ Die Tagesschausprecherin Judith Rakers kann als Ikone dieser Konjunktur des home farming gelten: Seit dem 22. März stolze Besitzerin der Henne „Schatzi“ und des Hahns „Giovanni“, versorgt sie ihre 136.0oo Follower auf Instagram regelmäßig mit „Chicken-Updates“ aus dem heiteren Hühnerleben. ${ }^{9}$

In der seit dem 19. Jahrhundert zu beobachtenden Geschichte des Haustiers stellt der coronabedingte Boom sicher nur eine kleine, wenn auch vielsagende Episode dar. Wenn im hochemotionalisierten Umgang mit dem Subjekt gewordenen Haustier eine Idee vom kindlichen Glück reproduziert wird, wie Clemens Wischermann mit Recht konstatier ${ }^{10}$, dann findet diese Kindheitskonstruktion derzeit ironischerweise im Homeoffice und damit unter dem Zeitregime häuslicher Präsenzarbeit statt. „Tierisch beste Freunde "11 kann es daher nur auf Zeit geben. Berichte über ausgesetzte CoronaHühner und einen „Hühner-Notstand“ in Berliner Tierheimen sprechen in dieser Hinsicht Bände. ${ }^{12}$ Zumindest mittelbar macht die ,Rache der Schuppentiere“ in Form einer Pandemie offenbar auch vor den Haustieren nicht Halt.

\section{Nutztiere}

Ganz besonders betroffen von dieser Rache scheinen jedoch die als Nutztiere etikettierten ehemaligen Wildtiere zu sein. Die in Dänemark im November 2020 angeordnete Vernichtung der gesamten Nerzpopulation ist hier stellvertretend für den Wunsch, durch die Tötung von Tieren auch die asymmetrische Herrschaftsbeziehung zwischen Menschen und Tieren wieder zu festigen, die durch zoonotische Pandemien regelrecht unterlaufen werden. Die bei Nerzen festgestellte sogenannte Cluster 5-Mutation des Coronavirus könne die Wirkung eines möglichen Impfstoffes minimieren, so die Befürchtung der dänischen Regierung. Zwar hat die epidemiologische Fachpresse inzwischen mitgeteilt, dass einerseits die Gefahr der Ansteckung durch das mutierte Virus sehr viel geringer sei als bei der unter Menschen grassierenden SARS-CoV-II-Variante und dass es andererseits keinerlei Nachweis für verminderte Wirkungen von Impfstoffen gäbe. Den Nerzen geht es dennoch an den Kragen. In ihnen wird ein „fortwährendes Reservoir auch für Infektionen beim Menschen" gesehen. ${ }^{13}$ Mit dieser spekulativen Wirkmacht als potenzielle Krankheitsverteiler wird somit einer möglichen ,Rache der Nerze präventiv entgegengetreten.

\section{References}

1. Wufei Yu, Coronavirus: Revenge of the Pangolins, New York Times, 5. März 2020 [https:/www.nytimes.com/2020/03/05/opinion/coronavirus-chinapangolins.html]. 
2. Joseph Goldstein, Bronx Zoo Tiger Is Sick With the Coronavirus, New York Times, 6. April 2020

[https://www.nytimes.com/2020/04/06/nyregion/bronx-zoo-tigercoronavirus.html].

3. CDC, Covid-19 and Animals [https://www.cdc.gov/coronavirus/2019ncov/daily-life-coping/animals.html].

4. [https://www.peta.de/menschenaffen-vor-corona-ansteckung-schuetzen-petafordert-schliessung-des]

5. Thomas Stillbauer, Langeweile im Zoo. Einsam in Corona-Zeiten: Tiere im Frankfurter Zoo vermissen die Besucher, Frankfurter Rundschau, 12. Juni 2020 [https://www.fr.de/frankfurt/tiere-frankfurter-vermissen-besucher-zr13649609.html].

6. Mieke Roscher und Anna-Katharina Wöbse, Zootiere während des Zweiten Weltkrieges. London und Berlin 1939-1945, in: WerkstattGeschichte 56 (2011), S. 46-62, 52.

7. z.B. Julia Fietz, Auf einmal Zeit für Hund und Katz. Haustiere und die Pandemie, Frankfurter Allgemeine Zeitung, 16.10.2020 [https://www.faz.net/aktuell/rhein-main/mehr-nachfrage-nach-haustieren-inder-corona-pandemie-17003928.html].

8. Titus Arnus, Ich wollt', ich hätt' ein Huhn, Süddeutsche Zeitung, 04.06.2020 [https://www.sueddeutsche.de/panorama/huehner-corona-nachfrage1.4926335].

9. [https://www.instagram.com/judith rakers/?hl=de].

10. Clemens Wischermann, Zwischen „Vieh“ und „Freund“. Historische Annäherungen an das Selbst eines Tieres, in: Tierisch beste Freunde. Über Haustiere und ihre Menschen, hg. v. Viktoria Krason, Christoph Willmitzer, Berlin 2017, S. 49-87.

11. Tierisch beste Freunde. Über Haustiere und ihre Menschen, hg. v. Viktoria Krason, Christoph Willmitzer, Berlin 2017.

12. Titus Arnu, Heimatlose Hühner, Süddeutsche Zeitung, 27.10.2020 [https://www.sueddeutsche.de/panorama/huehner-corona-tierheim1.5094334].

13. Christina Hohmann Jeddi, Coronavirus Variante aus Nerzen. Mutation wohl nicht so gefährlich Pharmakologische Zeitschrift, 18.11.2020 [https://www.pharmazeutische-zeitung.de/mutationen-wohl-nicht-sogefaehrlich-121853/].

SUGGESTED CITATION: Jaser, Christian; Roscher, Mieke: Covid 19. Eine tiergeschichtliche Annäherung an eine Pandemie, in: KWI-BLOG, [https://blog.kulturwissenschaften.de/covid-19/], 17.12.2020

DOI: $\underline{\text { https://doi.org/10.37189/kwi-blog/20201217-0830 }}$ 